\title{
Effect of Crystal Field and Spin-Orbit Coupling on Magnetic Susceptibility of Systems with $f^{2}$ Electron Con- figuration* $^{*}$
}

\author{
Charles M. Herzfeld and Daniel B. Levine
}

\begin{abstract}
The effects of a crystal field of octahedral symmetry and of spin-orbit coupling on an ion with $f^{2}$ electron configuration are considered. Only fourth-degree terms in the crystalfield potential are taken into account. The eigenvalues and eigenvectors of the resulting $33 \times 33$ secular determinant are determined numerically on the 704 electronic computer at the National Bureau of Standards for eight values of $D / \zeta$, where $D$ is a crystal-field parameter, and $\zeta$ the spin-orbit coupling constant of the ion. Perturbations by an external magnetic field are computed using second-order perturbation theory. All eigenvalues are tabulated for $D / \zeta=-10^{-4},-2 \times 10^{-4},-5 \times 10^{-4},-10^{-3},-2 \times 10^{-3},-5 \times 10^{-3},-10^{-2}$, $-10^{-1}$. They can be adapted directly to any finite nonzero positive value of $\zeta$ ' and, by interpolation, for values of $D / \zeta$ not tabulated. The magnetic susceptibility of a powder is calculated and displayed as a function of temperature and $D / \zeta$, for $\zeta=\infty, 10^{3} \mathrm{~cm}^{-1}$, and $5 \times 10^{2} \mathrm{~cm}^{-1}$. The relation of the results of the calculations to measurements on compounds of $U^{+4}$ is discussed.
\end{abstract}

\section{Introduction}

The magnetic and optical properties of ionic and atomic species in solids are affected considerably by their neighbors in the lattice. Crystal-field theory [1] ${ }^{1}$ furnishes a simple but reliable technique for taking such effects into account. In this paper are discussed the combined effects of the crystal field and the ionic spin-orbit coupling on the magnetic susceptibility of ions with an $f^{2}$ electron configuration. Examples of such systems are the $\mathrm{Nd}^{+3}\left(4 f^{2}\right)$ and $\mathrm{U}^{+4}$ $\left(5 f^{2}\right)$ salts; in the discussion the results are applied to some of the latter. Penney and Schlapp [2] have studied the properties of ions with $f^{2}$ configuration in a crystal field of cubic symmetry, with the assumption that the energy of spin-orbit coupling is very large (effectively infinite) compared to the crystalfield effect. They considered terms of fourth degree in the potential expansion, but neglected terms of sixth degree. Recently Hutchison and Candela [3] have treated the problem taking the sixth-degree terms in the potential into account, but they also assumed the effect of the ionic spin-orbit coupling to be large compared to the crystal-field splittings. The calculation reported in this paper makes no special assumption about the relative size of the crystal-field effect and the spin-orbit coupling, but neglects the sixth-degree terms in the potential expansion.

The Hamiltonian of an ion embedded in a crystal lattice, in the presence of an external magnetic field, may be written schematically in the form

$$
\mathrm{H}=\mathrm{H}_{0}+\zeta L \cdot S+\mathrm{H}_{c}+\mu \cdot H \text {. }
$$

Here $\mathrm{H}_{0}$ is the sum of three types of terms: The kinetic energy of the electrons of the ion, the potential

\footnotetext{
*This research was supported by the U. S. Air Force through the Air Force Office of Scientific Research of the Air Research and Development Command, under contract No. CSO and A 680-56-21. Reproduction in whole or in part is permitted for any purpose of the United States Government.
}

1 Figures in brackets indicate the literature references at the end of this paper. energy of the electrons in the field of the nucleus, and the energy of mutual repulsion of the electrons. The term proportional to $\zeta$ gives (approximately) the spin-orbit interaction of the electrons. $\mathrm{H}_{c}$ summarizes the perturbations of the electrons arising from the neighbors of the ion in the lattice, and $\mu \cdot H$ is the energy of the ion with magnetic moment $\mu$ in an external magnetic field $H$. The eigenvalues of $H$ are required for the calculation of the magnetic susceptibility.

\section{Eigenvalues of the Hamiltonian}

The form of the crystal-field energy $\mathrm{H}_{c}$ depends on the symmetry of the site of the ion. If this symmetry is cubic, $\dot{H}_{c}$ can be written in the form [1]

$$
\mathrm{H}_{c}=C+A \Sigma_{i}\left(x_{i}^{4}+y_{i}^{4}+z_{i}^{4}-(3 / 5) r_{i}^{4}\right)+\text { higher terms. }
$$

Here $C$ is a constant which can be disregarded for this calculation, $x_{i}, y_{i}$, and $z_{i}$ are the coordinates of the $i$ th electron (the nucleus of the ion is at the origin), $r_{i}=\left(x_{i}^{2}+y_{i}^{2}+z_{i}^{2}\right)^{1 / 2}$, and $A$ gives the magnitude and sign of the classical potential energy. Two cases of cubic symmetry are of interest: 1 . The ion is at the center of a regular octahedron, with negative charges located at the vertices. 2. The ion is at the center of a cube, with negative charges located at the vertices. In this calculation only the octahedral case is considered, where $A=(35 e \epsilon) /\left(4 r_{0}^{5}\right)$, with $e$ the electronic charge, $\epsilon$ the charge at a given vertex, and $r_{0}$ the distance from center to vertex.

The ground state of a free ion with $f^{2}$ electron configuration is ${ }^{3} H_{4}$, but since the effect of spin-orbit coupling is to be taken into account explicitly, the crystal-field calculation must involve all 33 states of the ${ }^{3} H$ Russell-Saunders level: ${ }^{3} H_{4},{ }^{3} H_{5}$, and ${ }^{3} H_{6}$. The most convenient representation is $S L M_{S} M_{L}$, with $S=1$ and $L=5$. The matrix for $\mathrm{H}_{c}+\zeta L \cdot S$ is written out explicitly in this representation, using matrix elements calculated from the formulas of 
Schlapp and Penney [4]. This matrix depends on two unknown parameters: $\zeta$, which gives the magnitude of the spin-orbit coupling, and $D$, the magnitude of the crystal-field perturbation. $D$ is proportional to $A$ of eq (2), and to $\left\langle r^{4}\right\rangle$, the average of $r^{4}$ for the $f$ electrons. These parameters are discussed below. The secular determinant of the $33 \times 33$ matrix so obtained can be factored into one $9 \times 9$ and three $8 \times 8$ determinants by rearranging rows and columns. The eigenvalues of the matrix are computed by solving for the roots of the factored secular determinants; the eigenvectors are also obtained. This computation was carried out on a 704 electronic computing machine, with a method due to Jacobi, adapted for machine use by Givens [5]. The eigenvalues are shown in figure 1 as a function of $D / \zeta$. The eigenvectors (which are also functions of $D / \zeta)$ are not shown, because the effect of their variation with $D / \zeta$ can be taken into account more efficiently in ways discussed below.

The matrix $\mu \cdot H$ is calculated in the $S L M_{S} M_{L}$ representation, and then transformed for each value of $D / \zeta$ to that representation in which $\mathrm{H}_{c}+\zeta L \cdot S$ is diagonal, using the eigenvector matrix of $\mathrm{H}_{c}+\zeta L \cdot S$ for the appropriate value of $D / \zeta$. Then the eigenvalues of $\mu \cdot H$ are calculated with second-order perturbation theory.

The eigenvalues of $\mathrm{H}_{c}+\zeta L \cdot S+\mu \cdot H$ are of the form $W=W_{c}+W_{1} H+W_{2} H^{2}$. Here $W_{c}$ is the eigenvalue of $\mathrm{H}_{c}+\zeta L \cdot S$, and $W_{1}$ and $W_{2}$ give the first-order and second-order contributions of $\mu \cdot H$. The values of $W_{c} / \zeta, W_{1} / \beta$, and $W_{2} \zeta / \beta^{2}$ are tabulated in table 1 , for the eight values of $D / \zeta$ for which the calculation has been carried out ( $\beta$ is the Bohr magneton). Each state is designated by a set $N$ of two quantum numbers $i$ and $j$; $i$ is a label for the irreducible representation of the cubic group [6] of the level $W_{c}$, and $j$ indicates the factor of the secular determinant in which the eigenvalue occurs. The original $33 \times 33$ secular determinant factors into four determinants: (One basis vector is given, to identify the determinants) $D_{1}:\left|M_{L}=3, M_{S}=-1\right\rangle, \mathrm{D}_{2}:|-5,-1\rangle, D_{3}: \mid-4$, $-1\rangle$, and $\left.D_{4}: 4,1\right\rangle . \quad D_{1}$ is a $9 \times 9$ determinant, the others are $8 \times 8$. It turns out that the different states belonging to a degenerate level $W_{c}$ arise always from different determinants, and further that the set of determinants from which they arise characterizes the irreducible representation of the octahedral group to which the level belongs [6]. Thus nondegenerate levels with $j=1$ belong to $\Gamma_{1}$, and those with $j=2$ belong to $\Gamma_{2}$. Doubly degenerate levels, always with $j=1$ and 2 belong, of course, to $\Gamma_{3}$. Triply degenerate levels with $j=1,3$, and 4 belong to $\Gamma_{4}$, those with $j=2,3$, and 4 to $\Gamma_{5}$. The method of assignment is simple. One $\Gamma_{1}$ state must be identified as such; the other assignments can be made by inspection, by noting whether any nonvanishing matrix elements of $\mu \cdot H$ connect $\Gamma_{1}$ with the unidentified

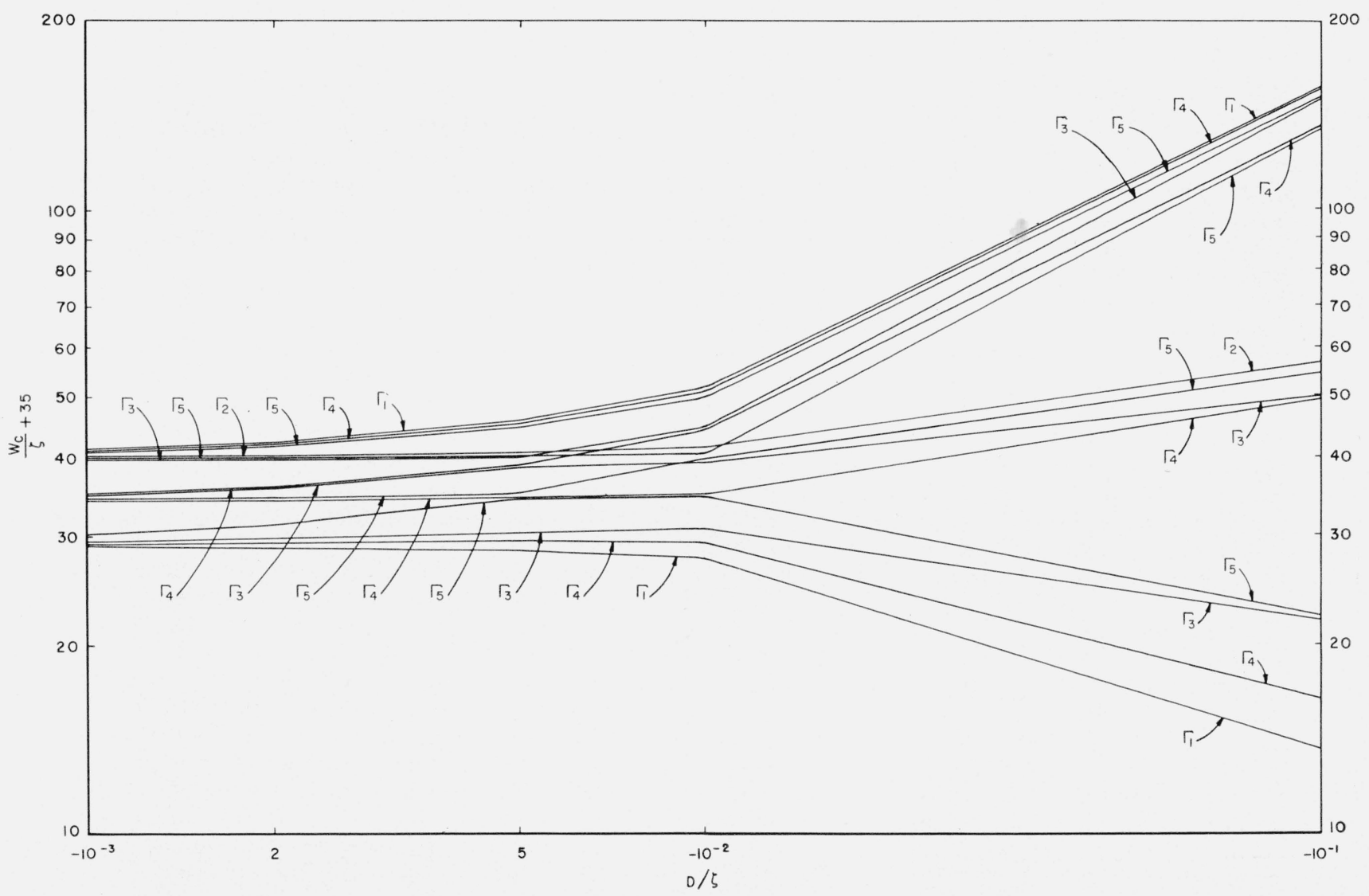

Figure 1. Eigenvalues $W_{c}$ of crystal-field and spin-orbit coupling as function of $D / \zeta$.

Calculations are made only for the values shown on abscissa. 
TABLe 1. Coefficients in expansion of energy levels: $W=W_{c}+W_{1} H+W_{2} H^{2}$

\begin{tabular}{|c|c|c|c|c|c|c|c|}
\hline \multicolumn{4}{|c|}{$D / \zeta=-10^{-4}$} & \multicolumn{4}{|c|}{$D / \zeta=-2 \times 10^{-4}$} \\
\hline$N$ & $W_{c} / \zeta$ & $W_{1} / \beta$ & $W_{2 \zeta / \beta^{2}}$ & $N$ & $W_{c} / \zeta$ & $W_{1} / \beta$ & $W_{2} \zeta / \beta^{2}$ \\
\hline $\begin{array}{l}1,1 \\
4,1 \\
4,3 \\
4,4 \\
3,1 \\
3,2\end{array}$ & $\begin{array}{l}-6.0138 \\
-5.9820 \\
-5.9820 \\
-5.9820 \\
-5.9592 \\
-5.9592\end{array}$ & $\begin{array}{l}0 \\
0 \\
-0.404 \\
\quad .404 \\
0 \\
0\end{array}$ & $\begin{array}{r}-133.0 \\
-125.0 \\
-12.4 \\
-12.4 \\
258.0 \\
-37.50\end{array}$ & $\begin{array}{l}1,1 \\
4,1 \\
4,3 \\
4,4 \\
3,1 \\
3,2\end{array}$ & $\begin{array}{l}-6.0276 \\
-5.9646 \\
-5.9646 \\
-5.9646 \\
-5.9194 \\
-5.9194\end{array}$ & $\begin{array}{l}0 \\
0 \\
-0.411 \\
0_{0}^{.411} \\
0\end{array}$ & $\begin{array}{c}-67.4 \\
-61.3 \\
-6.22 \\
-6.22 \\
129.0 \\
-18.8\end{array}$ \\
\hline $\begin{array}{l}5,2 \\
5,3 \\
5,4 \\
4,1 \\
4,3 \\
4,4\end{array}$ & $\begin{array}{r}-5.8901 \\
-5.8901 \\
-5.8901 \\
-0.9936 \\
-.9936 \\
-.9936\end{array}$ & $\begin{array}{c}0 \\
1.998 \\
-1.998 \\
0 \\
-3.013 \\
3.013\end{array}$ & $\begin{array}{r}37.31 \\
12.2 \\
12.2 \\
-25.5 \\
-253.0 \\
-253.0\end{array}$ & $\begin{array}{l}5,2 \\
5,3 \\
5,4 \\
4,1 \\
4,3 \\
4,4\end{array}$ & $\begin{array}{r}-5.7801 \\
-5.7801 \\
-5.7801 \\
-0.9872 \\
-.9872 \\
-.9872\end{array}$ & $\begin{array}{c}0 \\
1.999 \\
-1.999 \\
0 \\
-3.006 \\
3.006\end{array}$ & $\begin{array}{r}18.7 \\
6.05 \\
6.05 \\
-12.4 \\
-132.62 \\
-132.62\end{array}$ \\
\hline $\begin{array}{l}5,2 \\
5,3 \\
5,4 \\
3,1 \\
3,2\end{array}$ & $\begin{array}{r}-.9724 \\
-.9724 \\
-.9724 \\
-.9156 \\
-.9156\end{array}$ & $\begin{array}{l}0 \\
-2.587 \\
2.587 \\
0 \\
0\end{array}$ & $\begin{array}{r}-75.02 \\
221.0 \\
221.0 \\
-1490.0 \\
75.06\end{array}$ & $\begin{array}{l}5,2 \\
5,3 \\
5,4 \\
3,1 \\
3,2\end{array}$ & $\begin{array}{r}-.9454 \\
-.9454 \\
-.9454 \\
-.8303 \\
-.8303\end{array}$ & $\begin{array}{l}0 \\
-2.581 \\
2.581 \\
0 \\
0\end{array}$ & $\begin{array}{r}-37.0 \\
117.0 \\
117.0 \\
-789.0 \\
37.0\end{array}$ \\
\hline $\begin{array}{l}4,1 \\
4,3 \\
4,4 \\
3,1 \\
3,2\end{array}$ & $\begin{array}{r}-.9055 \\
-.9055 \\
-.9055 \\
4.9924 \\
4.9924\end{array}$ & $\begin{array}{l}0 \\
2.506 \\
-2.506 \\
0 \\
0\end{array}$ & $\begin{array}{r}1520.0 \\
31.8 \\
31.8 \\
-25.3 \\
-5759.6\end{array}$ & $\begin{array}{l}4,1 \\
4,3 \\
4,4 \\
3,1 \\
3,2\end{array}$ & $\begin{array}{r}-.8107 \\
-.8107 \\
-.8107 \\
4.9848 \\
4.9848\end{array}$ & $\begin{array}{l}0 \\
2.505 \\
-2.505 \\
0 \\
0\end{array}$ & $\begin{array}{r}801.0 \\
15.6 \\
15.6 \\
-1.34 \\
-2925.0\end{array}$ \\
\hline $\begin{array}{l}5,2 \\
5,3 \\
5,4 \\
2,2 \\
5,2 \\
5,3 \\
5,4\end{array}$ & $\begin{array}{l}\text { 4. } 9985 \\
4.9985 \\
4.9985 \\
5.0168 \\
5.0823 \\
5.0823 \\
5.0823\end{array}$ & $\begin{array}{c}0 \\
-0.696 \\
.696 \\
0 \\
0 \\
-2.224 \\
2.224\end{array}$ & $\begin{array}{c}5041.9 \\
-46.4 \\
-46.4 \\
623.82 \\
93.97 \\
-956.0 \\
-956.0\end{array}$ & $\begin{array}{l}5,2 \\
5,3 \\
5,4 \\
2,2 \\
5,2 \\
5,3 \\
5,4\end{array}$ & $\begin{array}{l}\text { 4. } 9971 \\
4.9971 \\
4.9971 \\
5.0336 \\
5.1652 \\
5.1652 \\
5.1652\end{array}$ & $\begin{array}{l}0 \\
-0.688 \\
\quad .688 \\
0 \\
0 \\
-2.230 \\
2.230\end{array}$ & $\begin{array}{r}2569.0 \\
-23.2 \\
-23.2 \\
309.0 \\
46.7 \\
-478.0 \\
-478.0\end{array}$ \\
\hline $\begin{array}{l}4,1 \\
4,3 \\
4,4 \\
1,1\end{array}$ & $\begin{array}{l}5.0994 \\
5.0994 \\
5.0994 \\
5.1146\end{array}$ & $\begin{array}{c}0 \\
-0.586 \\
0^{.586}\end{array}$ & $\begin{array}{r}-1165.0 \\
1000.0 \\
1000.0 \\
1190.0\end{array}$ & $\begin{array}{l}4,1 \\
4,3 \\
4,4 \\
1,1\end{array}$ & $\begin{array}{l}\text { 5. } 1992 \\
5.1992 \\
5.1992 \\
5.2291\end{array}$ & $\begin{array}{c}0 \\
-0.590 \\
0^{.590}\end{array}$ & $\begin{array}{r}-635.0 \\
501.0 \\
501.0 \\
636.0\end{array}$ \\
\hline
\end{tabular}

\begin{tabular}{|c|c|c|c|c|c|c|c|}
\hline \multicolumn{4}{|c|}{$\mathrm{D} / \zeta=-5 \times 10^{-4}$} & \multicolumn{4}{|c|}{$\mathrm{D} / \zeta=-10^{-3}$} \\
\hline$N$ & $W_{c / \zeta}$ & $W_{1} / \beta$ & $W_{2} \zeta / \beta^{2}$ & $N$ & $W_{c / \zeta}$ & $W_{1} / \beta$ & $W_{2} \zeta / \beta^{2}$ \\
\hline $\begin{array}{l}1,1 \\
4,1 \\
4,3 \\
4,4 \\
3,1 \\
3,2\end{array}$ & $\begin{array}{l}-6.0692 \\
-5.9158 \\
-5.9158 \\
-5.9158 \\
-5.8053 \\
-5.8053\end{array}$ & $\begin{array}{l}0 \\
0 \\
-0.4251 \\
\quad .4251 \\
0 \\
0\end{array}$ & $\begin{array}{c}-27.43 \\
-25.66 \\
-2.586 \\
-2.586 \\
52.91 \\
-7.670\end{array}$ & $\begin{array}{l}1,1 \\
4,1 \\
4,3 \\
4,4 \\
3,1 \\
3,2\end{array}$ & $\begin{array}{l}-6.1394 \\
-5.8451 \\
-5.8451 \\
-5.8451 \\
-5.6317 \\
-5.6317\end{array}$ & $\begin{array}{l}{ }_{0} \\
-0 \\
-0.449 \\
0_{0}^{.449} \\
0\end{array}$ & $\begin{array}{c}-14.08 \\
-13.09 \\
-1.37 \\
-1.37 \\
26.99 \\
-3.967\end{array}$ \\
\hline $\begin{array}{l}5,2 \\
5,3 \\
5,4 \\
4,1 \\
4,3 \\
4,4\end{array}$ & $\begin{array}{r}-5.4507 \\
-5.4507 \\
-5.4507 \\
-0.9682 \\
-.9682 \\
-.9682\end{array}$ & $\begin{array}{l}0 \\
2.000 \\
-2.000 \\
0 \\
-2.978 \\
2.978\end{array}$ & $\begin{array}{r}7.475 \\
2.405 \\
2.405 \\
-4.692 \\
-56.13 \\
-56.13\end{array}$ & $\begin{array}{l}5,2 \\
5,3 \\
5,4 \\
4,1 \\
4,3 \\
4,4\end{array}$ & $\begin{array}{r}-4.9024 \\
-4.9024 \\
-4.9024 \\
-0.9363 \\
-.9363 \\
-.9363\end{array}$ & $\begin{array}{c}0 \\
1.998 \\
-1.998 \\
0 \\
-2.935 \\
2.935\end{array}$ & $\begin{aligned} & 3.757 \\
& 1.18 \\
& 1.18 \\
&-2.122 \\
&-30.90 \\
&-30.90\end{aligned}$ \\
\hline $\begin{array}{l}5,2 \\
5,3 \\
5,4 \\
3,1 \\
3,2\end{array}$ & $\begin{array}{l}-.8685 \\
-.8685 \\
-.8685 \\
-.5695 \\
-.5695\end{array}$ & $\begin{array}{l}0 \\
-2.578 \\
2.578 \\
0 \\
0\end{array}$ & $\begin{array}{c}-14.09 \\
50.02 \\
50.02 \\
-335.9 \\
14.14\end{array}$ & $\begin{array}{l}5,2 \\
5,3 \\
5,4 \\
3,1 \\
3,2\end{array}$ & $\begin{array}{l}-.7527 \\
-.7527 \\
-.7527 \\
-.1203 \\
-.1203\end{array}$ & $\begin{array}{l}0 \\
-2.576 \\
2.576 \\
0 \\
0\end{array}$ & $\begin{array}{r}-6.546 \\
27.90 \\
27.90 \\
-198.0 \\
6.605\end{array}$ \\
\hline $\begin{array}{l}4,1 \\
4,3 \\
4,4 \\
3,1 \\
3,2\end{array}$ & $\begin{array}{r}-.5243 \\
-.5243 \\
-.5243 \\
4.9628 \\
4.9628\end{array}$ & $\begin{array}{l}0 \\
2.501 \\
-2.501 \\
0 \\
0\end{array}$ & $\begin{array}{r}340.7 \\
6.128 \\
6.128 \\
-4.931 \\
-1137.0\end{array}$ & $\begin{array}{l}4,1 \\
4,3 \\
4,4 \\
3,1 \\
3,2\end{array}$ & $\begin{array}{r}-.0421 \\
-.0421 \\
-.0421 \\
4.9280 \\
4.9280\end{array}$ & $\begin{array}{l}0 \\
2.492 \\
-2.492 \\
0 \\
0\end{array}$ & $\begin{array}{c}200.1 \\
2.99 \\
2.99 \\
-2.382 \\
-561.5\end{array}$ \\
\hline $\begin{array}{l}5,2 \\
5,3 \\
5,4 \\
2,2 \\
5,2 \\
5,3 \\
5,4\end{array}$ & $\begin{array}{l}\text { 4. } 9936 \\
\text { 4. } 9936 \\
\text { 4. } 9936 \\
\text { 5. } 0840 \\
\text { 5. } 4176 \\
\text { 5. } 4176 \\
\text { 5. } 4176\end{array}$ & $\begin{array}{c}0 \\
-0.6639 \\
\quad .6639 \\
0 \\
0 \\
-2.258 \\
2.258\end{array}$ & $\begin{array}{c}990.3 \\
-9.030 \\
-9.030 \\
129.1 \\
18.01 \\
-196.8 \\
-196.8\end{array}$ & $\begin{array}{l}5,2 \\
5,3 \\
5,4 \\
2,2 \\
5,2 \\
5,3 \\
5,4\end{array}$ & $\begin{array}{l}4.9900 \\
4.9900 \\
4.9900 \\
5.1680 \\
5.8490 \\
5.8490 \\
5.8490\end{array}$ & $\begin{array}{l}0 \\
-0.621 \\
\quad .621 \\
0 \\
0 \\
-2.300 \\
2.300\end{array}$ & $\begin{array}{c}485.8 \\
-4.33 \\
-4.33 \\
67.30 \\
8.557 \\
-103.0 \\
-103.0\end{array}$ \\
\hline $\begin{array}{l}4,1 \\
4,3 \\
4,4 \\
1,1\end{array}$ & $\begin{array}{l}5.5003 \\
5.5003 \\
5.5003 \\
5.5732\end{array}$ & $\begin{array}{l}0 \\
-0.5979 \\
0^{.5979}\end{array}$ & $\begin{array}{r}-257.1 \\
206.0 \\
206.0 \\
262.2\end{array}$ & $\begin{array}{l}4,1 \\
4,3 \\
4,4 \\
1,1\end{array}$ & $\begin{array}{l}6.0076 \\
6.0076 \\
6.0076 \\
6.1474\end{array}$ & $\begin{array}{c}0 \\
-0.614 \\
0^{.614}\end{array}$ & $\begin{array}{c}-134.4 \\
107.00 \\
107.00 \\
137.0\end{array}$ \\
\hline
\end{tabular}


TABle 1. Coefficients in expansion of energy levels: $W=W_{c}+W_{1} H+W_{2} H^{2}$ - Continued

\begin{tabular}{|c|c|c|c|c|c|c|c|}
\hline \multicolumn{4}{|c|}{$D / \zeta=-2 \times 10^{-3}$} & \multicolumn{4}{|c|}{$D / \zeta=-5 \times 10^{-3}$} \\
\hline$N$ & $W_{c} / \zeta$ & $W_{1} / \boldsymbol{\beta}$ & $W_{2} \zeta^{\prime} \beta^{2}$ & $N$ & $W_{c} / \zeta$ & $W_{1} / \boldsymbol{\beta}$ & $W_{2 \zeta} \zeta / \beta^{2}$ \\
\hline $\begin{array}{l}1,1 \\
4,1 \\
4,3 \\
4,4 \\
3,1 \\
3,2\end{array}$ & $\begin{array}{l}-6.2820 \\
-5.7382 \\
-5.7382 \\
-5.7382 \\
-5.3365 \\
-5.3365\end{array}$ & $\begin{array}{l}0 \\
0 \\
-0.504 \\
0 \\
0 \\
0\end{array}$ & $\begin{array}{l}-7.43 \\
-6.910 \\
-0.790 \\
-0.790 \\
14.1 \\
-2.15\end{array}$ & $\begin{array}{l}1,1 \\
4,1 \\
4,3 \\
4,4 \\
3,1 \\
3,2\end{array}$ & $\begin{array}{l}-6.722 \\
-5.616 \\
-5.616 \\
-5.616 \\
-4.7223 \\
-4.7223\end{array}$ & $\begin{array}{l}0 \\
0 \\
-0.7050 \\
.7050 \\
0 \\
0\end{array}$ & $\begin{array}{l}-3.262 \\
-3.271 \\
-0.5133 \\
-0.5133 \\
6.304 \\
-1.222\end{array}$ \\
\hline $\begin{array}{l}5,2 \\
5,3 \\
5,4 \\
4,1 \\
4,3 \\
4,4\end{array}$ & $\begin{array}{l}-3.8105 \\
-3.8105 \\
-3.8105 \\
-0.8705 \\
-.8705 \\
-.8705\end{array}$ & $\begin{aligned} & 0 \\
& 1.991 \\
&-1.991 \\
& 0 \\
&-2.830 \\
& 2.830\end{aligned}$ & $\begin{aligned} & 1.92 \\
& 0.555 \\
& 0.555 \\
&-0.873 \\
&-18.4 \\
&-18.4\end{aligned}$ & $\begin{array}{l}5,2 \\
5,3 \\
5,4 \\
4,1 \\
4,3 \\
4,4\end{array}$ & $\begin{array}{r}-0.7915 \\
-.7915 \\
-.7915 \\
-.6382 \\
-.6338 \\
-.6382\end{array}$ & $\begin{array}{c}0 \\
0.4043 \\
-.4043 \\
0 \\
-2.494 \\
2.494\end{array}$ & $\begin{array}{c}0.7243 \\
-14.86 \\
-14.86 \\
-0.2233 \\
2.200 \\
2.200\end{array}$ \\
\hline $\begin{array}{l}5,2 \\
5,3 \\
5,4 \\
3,1 \\
3,2\end{array}$ & $\begin{array}{r}-.5600 \\
-.5600 \\
-.5600 \\
.8211 \\
.8211\end{array}$ & $\begin{array}{l}0 \\
-2.571 \\
2.571 \\
0 \\
0\end{array}$ & $\begin{array}{r}-2.90 \\
17.0 \\
17.0 \\
-138.0 \\
2.94\end{array}$ & $\begin{array}{l}5,2 \\
5,3 \\
5,4 \\
3,1 \\
3,2\end{array}$ & $\begin{array}{l}.0147 \\
.0147 \\
.0147 \\
3.623 \\
3.623\end{array}$ & $\begin{array}{l}0 \\
-1.071 \\
1.071 \\
0 \\
0\end{array}$ & $\begin{array}{c}-0.8934 \\
12.34 \\
12.34 \\
-39.13 \\
-3.4767\end{array}$ \\
\hline $\begin{array}{l}4,1 \\
4,3 \\
4,4 \\
3,1 \\
3,2\end{array}$ & $\begin{array}{r}.9373 \\
.9373 \\
.9373 \\
4.8674 \\
4.8674\end{array}$ & $\begin{array}{l}0 \\
2.471 \\
-2.471 \\
0 \\
0\end{array}$ & $\begin{array}{r}138.0 \\
1.46 \\
1.46 \\
-1.09 \\
-276.0\end{array}$ & $\begin{array}{l}4,1 \\
4,3 \\
4,4 \\
3,1 \\
3,2\end{array}$ & $\begin{array}{l}3.946 \\
3.946 \\
3.946 \\
4.979 \\
4.979\end{array}$ & $\begin{array}{l}0 \\
2.380 \\
-2.380 \\
0 \\
0\end{array}$ & $\begin{array}{c}35.88 \\
0.5397 \\
.5397 \\
3.132 \\
-291.4\end{array}$ \\
\hline $\begin{array}{l}5,2 \\
5,3 \\
5,4 \\
2,2 \\
5,2 \\
5,3 \\
5,4\end{array}$ & $\begin{array}{l}\text { 4. } 9917 \\
\text { 4. } 9917 \\
\text { 4. } 9917 \\
\text { 5. } 3360 \\
\text { 6. } 7467 \\
\text { 6. } 7467 \\
6.7467\end{array}$ & $\begin{array}{l}0 \\
-0.543 \\
.543 \\
0 \\
0 \\
-2.375 \\
2.375\end{array}$ & $\begin{array}{c}236.0 \\
-1.97 \\
-1.97 \\
36.4 \\
3.89 \\
-55.9 \\
-55.9\end{array}$ & $\begin{array}{l}5,2 \\
5,3 \\
5,4 \\
2,2 \\
5,2 \\
5,3 \\
5,4\end{array}$ & $\begin{array}{l}5.069 \\
5.069 \\
5.069 \\
5.840 \\
9.628 \\
9.628 \\
9.628\end{array}$ & $\begin{array}{l}0 \\
-0.3325 \\
\quad .3325 \\
0 \\
0 \\
-2.5010 \\
2.5010\end{array}$ & $\begin{array}{c}277.1 \\
-0.5070 \\
-. .5070 \\
17.88 \\
1.256 \\
-26.82 \\
-26.82\end{array}$ \\
\hline $\begin{array}{l}4,1 \\
4,3 \\
4,4 \\
1,1\end{array}$ & $\begin{array}{l}\text { 7. } 0394 \\
\text { 7. } 0394 \\
\text { 7. } 0394 \\
\text { 7. } 2980\end{array}$ & $\begin{array}{c}0 \\
-0.630 \\
0 \\
0\end{array}$ & $\begin{array}{r}-72.7 \\
58.1 \\
58.1 \\
74.0\end{array}$ & $\begin{array}{l}4,1 \\
4,3 \\
4,4 \\
1,1\end{array}$ & $\begin{array}{l}10.228 \\
10.228 \\
10.228 \\
10.762\end{array}$ & $\begin{array}{c}0 \\
-0.6806 \\
0 . \\
0\end{array}$ & $\begin{array}{r}-35.21 \\
27.61 \\
27.61 \\
35.78\end{array}$ \\
\hline
\end{tabular}

\begin{tabular}{|c|c|c|c|c|c|c|c|}
\hline \multicolumn{4}{|c|}{$D / \zeta=-10^{-2}$} & \multicolumn{4}{|c|}{$D / \zeta=-10^{-1}$} \\
\hline$N$ & $W_{c} / \zeta$ & $W_{1} / \beta$ & $W_{2 \zeta / \beta^{2}}$ & $N$ & $W_{c} / \zeta$ & $W_{1} / \boldsymbol{\beta}$ & $W_{2} \zeta / \beta^{2}$ \\
\hline $\begin{array}{l}1,1 \\
4,1 \\
4,3 \\
4,4 \\
3,1 \\
3,2\end{array}$ & $\begin{array}{l}-7.4748 \\
-5.8051 \\
-5.8051 \\
-5.8051 \\
-4.1400 \\
-4.1400\end{array}$ & $\begin{array}{l}0 \\
0 \\
-1.0826 \\
1.0826 \\
0 \\
0\end{array}$ & $\begin{array}{c}-1.829 \\
-1.796 \\
-0.4688 \\
-.4688 \\
-3.305 \\
-1.252\end{array}$ & $\begin{array}{l}1,1 \\
4,1 \\
4,3 \\
4,4 \\
3,1 \\
3,2\end{array}$ & $\begin{array}{l}-21.447 \\
-18.68 \\
-18.68 \\
-18.68 \\
-13.228 \\
-13.228\end{array}$ & $\begin{array}{l}0 \\
0 \\
-2.289 \\
2.289 \\
0 \\
0\end{array}$ & $\begin{array}{r}-0.384 \\
-.012 \\
-.119 \\
-.119 \\
-.087 \\
-55.54\end{array}$ \\
\hline $\begin{array}{l}5,2 \\
5,3 \\
5,4 \\
4,1 \\
4,3 \\
4,4\end{array}$ & $\begin{array}{r}-0.3442 \\
-.3442 \\
-.3442 \\
-.1148 \\
-.1148 \\
-.1148\end{array}$ & $\begin{array}{r}0 \\
-2.706 \\
2.706 \\
0 \\
-1.972 \\
1.972\end{array}$ & $\begin{array}{c}-0.3923 \\
-20.34 \\
-20.34 \\
-0.0517 \\
19.86 \\
19.86\end{array}$ & $\begin{array}{l}5,2 \\
5,3 \\
5,4 \\
4,1 \\
4,3 \\
4,4\end{array}$ & $\begin{array}{r}-12.856 \\
-12.856 \\
-12.856 \\
\text { 14. } 374 \\
\text { 14. } 374 \\
14.374\end{array}$ & $\begin{array}{r}0 \\
-2.583 \\
2.583 \\
0 \\
-0.470 \\
.470\end{array}$ & $\begin{array}{c}55.19 \\
-0.064 \\
-.064 \\
.041 \\
-.872 \\
-.872\end{array}$ \\
\hline $\begin{array}{l}3,1 \\
3,2 \\
5,2 \\
5,3 \\
5,4\end{array}$ & $\begin{array}{l}\text { 4. } 2067 \\
\text { 4. } 2067 \\
\text { 4. } 8578 \\
\text { 4. } 8578 \\
\text { 4. } 8578\end{array}$ & $\begin{array}{l}0 \\
0 \\
0 \\
1.454 \\
-1.454\end{array}$ & $\begin{array}{c}-0.1733 \\
-22.78 \\
4.865 \\
-0.6913 \\
-.6913\end{array}$ & $\begin{array}{l}3,1 \\
3,2 \\
5,2 \\
5,3 \\
5,4\end{array}$ & $\begin{array}{l}\text { 14. } 81 \\
14.81 \\
19.424 \\
19.424 \\
19.424\end{array}$ & $\begin{array}{l}0 \\
0 \\
0 \\
-0.143 \\
.143\end{array}$ & $\begin{array}{r}0.216 \\
-1.891 \\
-3.865 \\
0.972 \\
.972\end{array}$ \\
\hline $\begin{array}{l}5,2 \\
5,3 \\
5,4 \\
2,2\end{array}$ & $\begin{array}{l}\text { 5. } 5857 \\
\text { 5. } 5857 \\
\text { 5. } 5857 \\
\text { 6. } 6800\end{array}$ & $\begin{array}{c}0 \\
0.3332 \\
-.3332 \\
0\end{array}$ & $\begin{array}{c}6.340 \\
0.8526 \\
.8526 \\
12.03\end{array}$ & $\begin{array}{l}2,2 \\
5,2 \\
5,3 \\
5,4\end{array}$ & $\begin{array}{l}21.8 \\
99.38 \\
99.38 \\
99.38\end{array}$ & $\begin{array}{l}0 \\
0 \\
0.427 \\
-.427\end{array}$ & $\begin{aligned} & 6.010 \\
& 0.0443 \\
&-1.46 \\
&-1.46\end{aligned}$ \\
\hline $\begin{array}{l}4,1 \\
4,3 \\
4,4 \\
3,1 \\
3,2\end{array}$ & $\begin{array}{l}\text { 9. } 0480 \\
9.0480 \\
9.0480 \\
9.6933 \\
9.6933\end{array}$ & $\begin{array}{l}\quad{ }^{2} \\
\text { 2. } 247 \\
-2.247 \\
0 \\
0\end{array}$ & $\begin{array}{c}-22.92 \\
0.2985 \\
0.2985 \\
23.06 \\
0.6781\end{array}$ & $\begin{array}{l}4,1 \\
4,3 \\
4,4 \\
3,1 \\
3,2\end{array}$ & $\begin{array}{l}100.483 \\
100.483 \\
100.483 \\
114.018 \\
114.018\end{array}$ & $\begin{array}{l}0 \\
1.426 \\
-1.426 \\
0 \\
0\end{array}$ & $\begin{array}{r}-0.966 \\
.874 \\
.874 \\
-0.004 \\
-.0716\end{array}$ \\
\hline $\begin{array}{l}5,2 \\
5,3 \\
5,4 \\
4,1 \\
4,3 \\
4,4 \\
1,1\end{array}$ & $\begin{array}{l}14.741 \\
14.741 \\
14.741 \\
15.712 \\
15.712 \\
15.712 \\
16.555\end{array}$ & $\begin{array}{l}0 \\
-2.582 \\
2.582 \\
0 \\
-0.6925 \\
\quad .6925 \\
0\end{array}$ & $\begin{array}{r}.5104 \\
-15.99 \\
-15.99 \\
-21.92 \\
16.48 \\
16.48 \\
22.33\end{array}$ & $\begin{array}{l}5,2 \\
5,3 \\
5,4 \\
4,1 \\
4,3 \\
4,4 \\
1,1\end{array}$ & $\begin{array}{l}115.452 \\
115.452 \\
115.452 \\
119.223 \\
119.223 \\
119.223 \\
121.247\end{array}$ & $\begin{array}{c}0 \\
-1.206 \\
1.206 \\
0 \\
-0.161 \\
.161 \\
0\end{array}$ & $\begin{array}{l}\quad .1186 \\
-2.00 \\
-2.00 \\
-6.65 \\
2.67 \\
2.67 \\
7.67\end{array}$ \\
\hline
\end{tabular}


level. The lowest lying level belongs to $\Gamma_{1}$ and arises from $D_{1}$. Matrix elements for $\mu \cdot H$ that connect any eigenvector of $D_{1}$ with any vector of the matrices of the other determinants must vanish. Therefore only levels with $j=1$ are thus connected to $\Gamma_{1}$, and hence levels with $j=1,3$, and 4 belong to $\Gamma_{4}$. Similarly for all other levels.

The results in table I cover a wide range of situations, and allow one to calculate magnetic properties quite readily. The method of presentation of the results permits their adaptation in two ways. First, the eigenvalues can be adapted to any finite nonzero value of the spin-orbit coupling constant $\zeta$. For positive $\zeta$ the problem corresponds to the octahedral case with $f^{2}$ configuration. (For negative $\zeta$ the problem corresponds to the cubic case with $f^{12}$ configuration.) The adaptation is straightforward. Thus for $D / \zeta=$ $-10^{-4}$ and for the state $N=2,3, W_{c} / \zeta=-5.9820$, $W_{1} / \beta=-0.404, \quad W_{2} \zeta / \beta^{2}=-12.4$. Hence $W_{c}=$ $-5.9820 \zeta, W_{1}=-0.404 \beta, W_{2}=-12.4 \beta^{2} / \zeta$. If $\zeta=10^{3}$ $\mathrm{cm}^{-1}, W_{c}=-5.9820 \times 10^{3} \mathrm{~cm}^{-1}, W_{1}=-0.404 \beta, W_{2}=$ $-12.4 \beta^{2} \times 10^{-3}$. The second adaptation involves interpolation of the results for values of $D / \zeta$ other than those covered here. This can be accomplished by a graphical interpolation of the values in table I. These vary sufficiently slowly to permit interpolation of $W_{c}, W_{1}$, and $W_{2}$ for each state.

\section{Calculated Magnetic Susceptibility and Discussion of the Results}

The magnetic susceptibility per mole, $\chi_{M}$, of ions with $f^{2}$ configuration was calculated [7] for a powder, as a function of temperature and $D$. The results are plotted as $\chi^{-1}$ in figure 2 . The results for our calculation for $\zeta=10^{3} \mathrm{~cm}^{-1}$, and $\zeta=5 \times 10^{2} \mathrm{~cm}^{-1}$ are given for several values of $D$. Also some results of the theory of Penney and Schlapp [2] (effectively $\zeta=\infty)$ are shown. For $D / \zeta=-10^{-1},-10^{-2}$, and

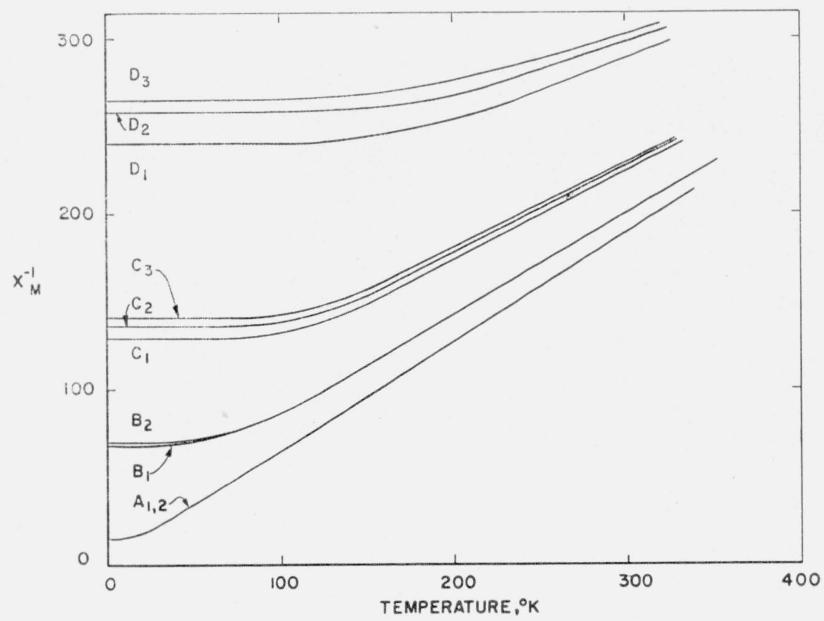

Figure 2. $\chi^{-1}$ versus temperature.

Curves $A_{k}$ for $D=-0.1 \mathrm{~cm}^{-1} ; B_{k}$ for $D=-0.5 \mathrm{~cm}^{-1} ; C_{k}$ for $D=-1.0 \mathrm{~cm}^{-1}$; and $D_{k}$ for $D=-2.0 \mathrm{~cm}^{-1}$. $k=1$ corresponds to $\zeta=500 \mathrm{~cm}^{-1}, k=2$ to $\zeta=10^{3} \mathrm{~cm}^{-1}$. Curves $C_{3}$ and $D_{3}$ are taken from Penney and Schlapp theory $(\zeta=\infty)$, and crystal-field splittings comparable to $C_{2}$ and $D_{2}$, respectively.
$-5 \times 10^{-3}$, with $\zeta=10^{3} \mathrm{~cm}^{-1}, \chi^{-1}$ is practically independent of temperature up to $300^{\circ} \mathrm{K}$, and equals $5,102,1,080$, and 602 , respectively.

The matrix elements of $\mathrm{H}_{c}+\zeta \dot{L} \cdot S$ are given [2] in terms of the two undetermined parameters $\zeta$ and $D$. The value of $\zeta$ in a solid is uncertain, though the free ion value is often a useful first approximation. The correct value of $\zeta$ for $U^{+4}$ lies probably [3] between $600 \mathrm{~cm}^{-1}$ and $800 \mathrm{~cm}^{-1}$. Thus the calculated susceptibilities reported in this paper bracket the most likely value. Direct calculation, using eigenstates $\left|f^{2}{ }^{3} H M_{S} M_{L}\right\rangle$ obtained by standard methods [8] shows for the regular octahedron that the parameter $D=-\sqrt{2 \pi} \ell \epsilon\left\langle r^{4}\right\rangle /\left(9 r_{0}^{5}\right)$, indicating that $D$ is negative.

The calculations reported in this paper agree in a general way with the measurements and calculations of Hutchison and Candela [3] on $\left[\left(\mathrm{CH}_{3}\right)_{4} \mathrm{~N}\right]_{2} \mathrm{U} \mathrm{Cl}_{6}$. They find a value of $\chi_{M}^{-1}=520$, which is independent of temperature over the range measured, and explain this satisfactorily with their theory. Because the calculations reported in this paper always give a nondegenerate groundstate $\left(\Gamma_{1}\right)$, they also give a temperature independent $\chi_{M}^{-1}$ for sufficiently large crystal-field splittings, and this for any value of $\zeta$. The calculations reported in this paper shed some light on other measurements [9] of powder susceptibilities of compounds containing $\mathrm{U}^{+4}$. These measure ments (on $\mathrm{U}\left(\mathrm{SO}_{4}\right)_{2} 4 \mathrm{H}_{2} \mathrm{O}, \mathrm{U}\left(\mathrm{C}_{2} \mathrm{O}_{4}\right)_{2} 6 \mathrm{H}_{2} \mathrm{O}, \mathrm{UF}_{4} \mathrm{NaF}$, $(\gamma) \mathrm{UF}_{4} 2 \mathrm{NaF}$, and $\mathrm{UF}_{4} 3 \mathrm{NaF}$ ) cannot be explained by a theory of the Penney and Schlapp type (i. e., $\zeta=\infty)$, nor by the present theory, though the present calculations somewhat improve agreement with the measurements on $\mathrm{U}\left(\mathrm{C}_{2} \mathrm{O}_{4}\right) 6 \mathrm{H}_{2} \mathrm{O}$. This indicates that the finite value of $\zeta$ does not play a very important role in these systems, and that a more realistic crystal-field potential, involving terms of second, fourth, and sixth degree, is required for an understanding of these systems.

The computation of the eigenvalues $W$ and the eigenvectors was carried out on a 704 computer by Miss Irene Stegun of the Computation Section of the Applied Mathematics Division of the National Bureau of Standards. Her help was essential for this study, and is gratefully acknowledged.

\section{References}

[1] B. Bleaney and K. W. H. Stevens, Repts. Progr. Phys. 16, 108 (1953)

[2] W. G. Penney and R. Schlapp, Phys. Rev. 41, 194 (1932).

[3] C. A. Hutchison, Jr., and G. Candela, J. Chem. Phys. 27, 707 (1957).

[4] R. Schlapp and W. G. Penney, Phys. Rev. 42, 666 (1932).

¿5] W. Givens, Numerical computation of the characteristic values of a real symmetric matrix, Oak Ridge Report ORNL 1574 (1954)

[6] H. Bethe, Ann. Phys. 3, 133 (1929).

[7] J. H. Van Vleck, The theory of electric and magnetic susceptibilities, ch. VII (Oxford University Press, New York, N. Y., 1932).

[8] E. U. Condon and G. H. Shortley, The theory of atomic spectra, ch. 8 (Cambridge University Press, New York, N. Y., 1935)

[9] C. A. Hutchison, Jr., and C. M. Herzfeld, J. Chem. Phys. 23, 1650 (1950).

Washington, April 18, 1958. 